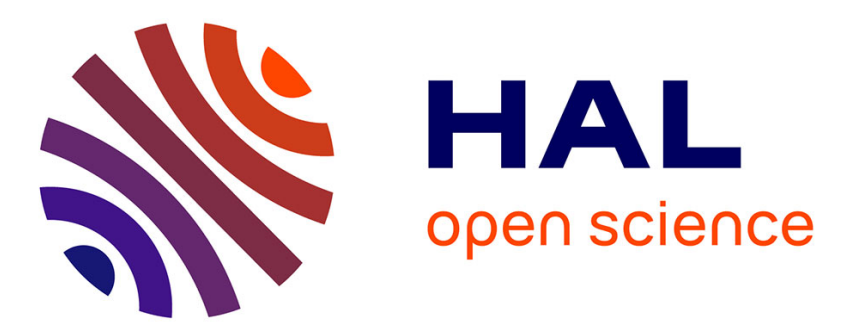

\title{
Le bien-être à l'école et au collège selon les élèves et les chefs d'établissements : des représentations différenciées
}

Tiphaine Gaudonville, Séverine Ferrière, Philippe Guimard, Agnès Florin, Fabien Bacro

\section{- To cite this version:}

Tiphaine Gaudonville, Séverine Ferrière, Philippe Guimard, Agnès Florin, Fabien Bacro. Le bien-être à l'école et au collège selon les élèves et les chefs d'établissements : des représentations différenciées. Recherches \& éducations, 2017. halshs-02302457

\section{HAL Id: halshs-02302457 \\ https://shs.hal.science/halshs-02302457}

Submitted on 1 Oct 2019

HAL is a multi-disciplinary open access archive for the deposit and dissemination of scientific research documents, whether they are published or not. The documents may come from teaching and research institutions in France or abroad, or from public or private research centers.
L'archive ouverte pluridisciplinaire HAL, est destinée au dépôt et à la diffusion de documents scientifiques de niveau recherche, publiés ou non, émanant des établissements d'enseignement et de recherche français ou étrangers, des laboratoires publics ou privés. 


\section{Recherches \& éducations}

17 | Juin 2017

Le bien-être à l'école: un processus de production du bien-être? Tome 1

\section{Le bien-être à l'école et au collège selon les élèves et les chefs d'établissements : des représentations différenciées}

Tiphaine Gaudonville, Séverine Ferrière, Philippe Guimard, Agnès Florin et Fabien Bacro

\section{OpenEdition}

Journals

Édition électronique

URL : http://journals.openedition.org/rechercheseducations/3642

ISSN : $1760-7760$

Éditeur

Société Binet Simon

Édition imprimée

Date de publication : 17 juin 2017

ISSN : 1969-0622

Ce document vous est offert par L'Université de Nantes

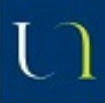

UNIVERSITÉ DE NANTES

\section{Référence électronique}

Tiphaine Gaudonville, Séverine Ferrière, Philippe Guimard, Agnès Florin et Fabien Bacro, « Le bien-être

à l'école et au collège selon les élèves et les chefs d'établissements : des représentations différenciées », Recherches \& éducations [En ligne], 17 | Juin 2017, mis en ligne le 09 janvier 2019, consulté le 01 octobre 2019. URL : http://journals.openedition.org/rechercheseducations/3642

Ce document a été généré automatiquement le 1 octobre 2019.

Propriété intellectuelle 


\title{
Le bien-être à l'école et au collège selon les élèves et les chefs d'établissements : des représentations différenciées
}

\author{
Tiphaine Gaudonville, Séverine Ferrière, Philippe Guimard, Agnès Florin \\ et Fabien Bacro
}

\section{Introduction}

1 Les recherches sur le bien-être à l'école ont émergé dans un contexte marqué par une réorientation des finalités de l'école. Aujourd'hui, les objectifs éducatifs ne visent plus seulement le développement des connaissances et des savoirs des enfants. Ils concernent également l'épanouissement de l'enfant à l'école afin qu'il s'intègre le mieux possible dans la société et qu'il réalise ainsi ses potentialités et vive pleinement sa vie (Gill, 2009 ; Guimard, Bacro, \& Florin, 2014). La thématique du bien-être à l'école, dans le champ de la psychologie, apparait ainsi comme un nouvel objet d'étude à travailler en tant que tel, en complément des thématiques plus traditionnelles centrées sur les facteurs explicatifs des résultats académiques des élèves.

Toutefois, en psychologie la définition du bien-être en contexte scolaire ne fait pas consensus, de nombreux indicateurs étant couramment utilisés pour l'évaluer (Pollar \& Lee, 2003). Les chercheurs s'accordent cependant sur plusieurs points : d'une part, les élèves sont les plus à mêmes d'évaluer leur bien-être, d'autre part le bien-être à l'école est une dimension importante de leur qualité de vie; enfin celui-ci renvoie à l'évaluation cognitive et affective de la satisfaction globale des expériences vécues à l'école (Huebner, Ash, \& Laughlin, 2001). Dans ce cadre, le bien-être perçu est appréhendé par deux catégories d'instruments selon qu'ils évaluent un construit unidimensionnel ou multidimensionnel (Randolph, Kangas, \& Ruokamo, 2009 ; Long et al., 2012). Les mesures unidimensionnelles qui appréhendent un niveau global de satisfaction des élèves sont les plus utilisées. Les mesures multidimensionnelles, elles, rendent compte du bien-être 
comme la résultante d'un ensemble de dimensions spécifiques: le climat de la classe perçu, le sentiment de compétence perçu, etc. (Randolph, Kangas, \& Ruokamo, 2009; 2010). Néanmoins, les dimensions constitutives du bien-être ne sont pas stabilisées et sont susceptibles de varier selon l'âge et l'évolution psychologique des enfants (Currie et al., 2012) ou selon les caractéristiques des systèmes scolaires ou des établissements fréquentés (Afsa, 2015 ; Billaudeau \& Vercambre-Jacquot, 2015 ; Guimard et al., 2015).

S'agissant des études internationales effectuées sur ce thème, divers travaux indiquent que plusieurs catégories de facteurs (individuels, sociaux, émotionnels, etc.) peuvent influencer la satisfaction des élèves à l'école (Hoffman, 2009; Randolph, Kangas, \& Ruokamo, 2009, 2010 ; Zullig et al., 2009). Ainsi, les filles sont généralement plus satisfaites de l'école que les garçons et les élèves les plus jeunes ont tendance à être plus satisfaits que leurs aînés. Eccles et Roeser (2011) montrent que cette diminution de la satisfaction scolaire s'opère en particulier lors de la transition école primaire-collège. Par ailleurs, les écoliers se sentent plus soutenus par leurs enseignants que les collégiens (MurrayHarvey, 2010), le soutien des enseignants perçu par les élèves étant l'une des dimensions les plus prédictives du niveau de bien-être et de la réussite à l'école (Verkuyten \& Thijs, 2002). Par ailleurs, les collégiens déclarent un niveau d'anxiété par rapport aux apprentissages plus élevé que celui des écoliers. D’autres études révèlent également que la qualité des relations aux pairs et aux adultes de l'école influence la réussite, la satisfaction scolaire et le bien-être futur (Epstein, 1981; Wigfield et al., 2006; Eccles \& Roeser, 2011 ; OCDE, 2015). Notons toutefois que ces résultats portent davantage sur les élèves de collège que d'école primaire et qu'ils sont issus d'études transversales apportant des résultats limités par rapport aux études longitudinales.

Signalons enfin, qu'en France, les recherches sur le bien-être à l'école sont rares. Néanmoins, quelques enquêtes mettent en évidence une appréciation négative de l'école des élèves français. L'étude réalisée pour l'Unicef-France (Debardieux, 2011) révèle que $28 \%$ des écoliers et des collégiens n'aiment pas trop ou pas du tout aller à l'école. Ces chiffres sont confirmés par l'enquête de l'Association de la fondation étudiante pour la ville (Bavoux \& Pugin, 2013) sur une population spécifique de son champ d'intervention (socialement plutôt défavorisée), puisque $45 \%$ des écoliers et collégiens déclarent aimer « un peu » aller à l'école » et $30 \%$ " pas trop ou pas du tout ». On observe une différence entre l'école où $60 \%$ des écoliers aiment « beaucoup » aller à l'école et le collège où ils ne sont plus que $16 \%$. Par ailleurs, l'enquête internationale HBSC 2010 montrait déjà un classement particulièrement bas des élèves français quant à leur stress ressenti pour le travail scolaire et le soutien social perçu à l'école, comparativement aux élèves des autres pays (Currie et al., 2012). Ces données rejoignent celles de PISA 2012 montrant que $47 \%$ des élèves français déclarent se sentir chez eux à l'école, ce qui est bien en dessous de la moyenne des pays de l'OCDE (OCDE, 2013). Le sentiment d'appartenance des élèves français à l'école est également le plus faible des pays de l'OCDE et leur niveau d'anxiété dans l'apprentissage des mathématiques est très élevé (OCDE, 2013).

\section{Objectifs de l'étude}

2 Bien qu'elles s'avèrent nécessaires pour améliorer le développement personnel et la réussite scolaire des élèves, les actions destinées à favoriser le bien-être à l'école et ayant fait l'objet d'une évaluation scientifique rigoureuse sont rares (voir cependant Durlak et al., 2011; Shankland \& Rosset, 2016) ou difficiles à identifier compte tenu de la variabilité 
des définitions du bien-être. Situé dans cette perspective, cet article se propose de repérer des domaines susceptibles de promouvoir le bien-être à l'école en croisant le point de vue des élèves et des chefs d'établissements dans lesquels ils sont scolarisés. Ces préconisations s'appuient sur les données d'une étude longitudinale de deux années qui nous a conduits à valider un outil d'évaluation multidimensionnel de bien-être auprès d'élèves d'écoles primaires et de collèges (Guimard et al., 2015) ${ }^{1}$. Rarement adoptée dans les études sur le bien-être, cette démarche longitudinale permet d'examiner l'évolution du bien-être perçu des élèves, en particulier lors du passage école-collège qui introduit des modifications importantes dans la vie des élèves (Lester \& Cross, 2015) et d'identifier les dimensions du bien-être les moins satisfaisantes. Par ailleurs, ces préconisations tiennent compte des représentations du bien-être des chefs d'établissements, telles que renseignées dans le cadre d'entretiens individuels.

\section{Méthodologie}

\section{Participants}

3 Cette étude longitudinale de deux années (T1 et T2) porte sur un échantillon de 557 élèves (301 filles et 256 garçons) scolarisés à l'école primaire $(\mathrm{N}=253)$ et au collège $(\mathrm{N}=354)$. Ils sont âgés en moyenne de 11,12 ans (ET : 1,65) et scolarisés dans 8 écoles et 12 collèges de Nantes et de sa région. $66,2 \%$ des élèves appartiennent à des établissements publics, $13,3 \%$ à des établissements publics situés en ZEP et $20,5 \%$ à des établissements privés. Cette répartition est globalement assez proche de la répartition nationale (Public: $60 \%$; Public ZEP : $20 \%$; Privé : $20 \%$ ). Par ailleurs, 115 élèves scolarisés en CM2 lors de l'année 1 ont pu être suivis en 6ème lors de l'année 2.

En parallèle, des entretiens ont été réalisés auprès de 17 chefs d'établissements (10 en école primaire et 7 en collège), de ZEP, non ZEP et du privé sous contrat (tableau 1). Les objectifs étaient notamment d'identifier leurs représentations du bien-être et les actions en faveur du bien-être conduites dans leur établissement. 
Tableau 1 : caractéristiques des chefs d'établissements ayant accordés un entretien

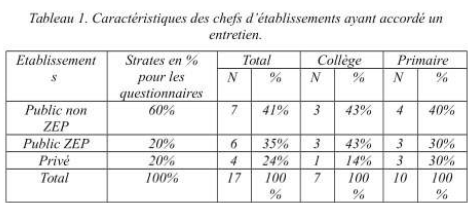

\section{Protocole d'évaluation auprès des élèves et procédure}

Annexe 1. Echelle de satisfaction scolaire globale (Huebner et al., 2012 ; Guimard et al., 2015)

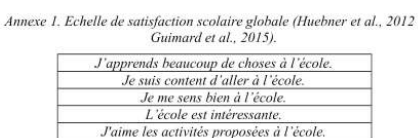


4 La satisfaction scolaire globale des élèves a été évaluée avec une échelle unidimensionnelle incluse dans la version abrégée de la « Multidimensional Sudents Life Satisfaction Scale» (MSLSS) de Huebner et al. (2012) (annexe 1). Les élèves devaient choisir leurs réponses sur une échelle de 5 items en 6 points allant de 1 (Absolument pas d'accord) à 6 (Absolument d'accord). Le niveau de satisfaction scolaire des élèves a été évalué en calculant la moyenne des scores obtenus aux 5 items. Un score élevé reflète un haut niveau de satisfaction à l'égard de l'école.

Annexe 2. Questionnaire Be-Scol (Guimard et al., 2015)

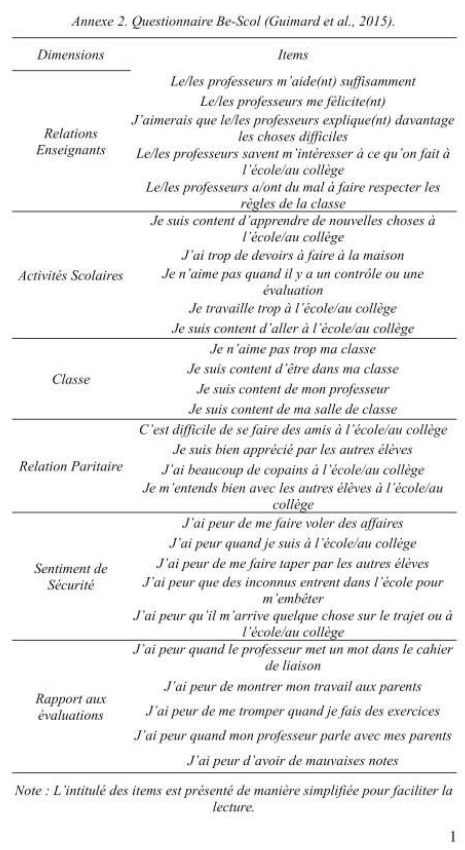

5 Cette échelle multidimensionnelle de bien-être à l'école est composée de 28 items dont le format de présentation est inspiré du Self Perception Profile de Harter (1982), traduit et validé par Pierrehumbert et al. (1987). Pour chaque item, deux types d'élèves sont présentés et l'enfant doit choisir ceux auxquels il ressemble le plus. On lui demande ensuite de nuancer sa réponse en indiquant si les élèves qu'il a choisis sont vraiment ou à peu près comme lui. Les réponses se distribuent sur une échelle en 4 points, un score de 4 correspondant toujours à l'appréciation la meilleure. Ce questionnaire a été construit sur la base des données de la littérature internationale et de nos propres travaux sur le bienêtre et la qualité de vie des enfants (Bacro et al., 2011 ; Bacro et al., 2013 ; Ferrière, 2013 ; Guimard et al., 2013; Guimard, Bacro, Florin, 2014). L'objectif de cette échelle est d'appréhender 6 dimensions de la vie scolaire : les activités scolaires, les relations avec les enseignants, la satisfaction à l'égard de la classe, les relations paritaires, le sentiment de sécurité et le rapport aux évaluations (annexe 2). Pour chacune d'entre elles, le niveau de bien-être des élèves est évalué en calculant la moyenne des scores obtenus aux items qui la composent. L'étude de validation réalisée sur les données de l'année 1 (Guimard et al., 2015) montre que l'échelle présente des propriétés métriques tout à fait acceptables en 
termes de structure factorielle, de validité convergente et divergente, de cohérence interne et de fidélité test-restest.

\section{Procédure}

6 Après avoir obtenu le consentement des enfants et de leurs parents, les données ont été recueillies en mars/avril de chaque année par des étudiantes en master de psychologie. D'une durée de deux fois quarante-cinq minutes environ, les passations ont été réalisées collectivement, en classe ou dans une salle de l'établissement.

\section{Entretiens auprès des chefs d'établissement}

7 Nous avons sollicité les chefs d'établissements ayant participé à la phase d'enquête auprès des élèves pour la réalisation d'un entretien semi-direct. La consigne initiale identique pour les chefs d'établissement en primaire et en collège, sollicitait l'avis de ces professionnels sur ce que recouvre la notion de bien-être, au sens large, dans leur établissement. La grille d'entretien était composée de 4 axes principaux: les caractéristiques générales de l'établissement et l'équipe éducative ; les représentations de la notion de bien-être (des élèves, du personnel) ; les actions identifiées comme relevant du bien-être, leur évaluation, ainsi que les freins éventuels; les perspectives au sein de l'établissement en matière de bien-être.

\section{Résultats}

Dans une première partie sont présentés les résultats des analyses quantitatives permettant d'évaluer le degré de bien-être des élèves dans les six dimensions du questionnaire et d'apprécier son évolution sur les deux années. La seconde partie présente l'étude qualitative du discours des chefs d'établissements en vue d'identifier leurs représentations du bien-être.

\section{Evolution du bien-être à l'école perçu par les élèves}


Tableau 2. Evolution du bien-être entre $\mathrm{T} 1$ et $\mathrm{T} 2$ sur l'ensemble de l'échantillon $(\mathrm{N}=557)$ et en fonction du type d'établissement (école primaire vs collège) $(\mathrm{N}=442)$

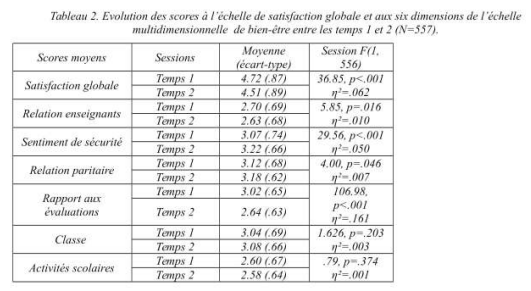

Afin d'apprécier l'évolution du bien-être des élèves entre les deux temps de mesure sur l'ensemble de l'échantillon et en fonction du type d'établissement (école primaire vs collège), des analyses statistiques (ANOVA à mesures répétées) ont été réalisées sur les scores à l'échelle de satisfaction globale et aux 6 dimensions du questionnaire de bienêtre (tableaux 2 et 3), à l'aide du logiciel SPSS 21 (seuil de significativité de $5 \%$ retenu). Pour l'ensemble de l'échantillon, on constate tout d'abord, que le score global de satisfaction scolaire a significativement diminué entre $\mathrm{T} 1$ et $\mathrm{T} 2(4.72$ et $4.51, \mathrm{~F}(1,556)=$ $36.85, \mathrm{p}<.001)$. Par ailleurs, en ce qui concerne les six dimensions au questionnaire de bien-être, les scores ont diminué pour les dimensions « relations avec les enseignants » $(\mathrm{F}(1,556)=5.85, \mathrm{p}=.016)$ et "rapport aux évaluations » $(\mathrm{F}(1,556)=106.98, \mathrm{p}<.001)$. En revanche, les scores sur les dimensions «sentiment de sécurité » et "relations paritaires" ont augmenté significativement entre les deux temps de mesure (respectivement F $(1,556)=9.42, p<.001$ et $F(1,556)=4.00, p=.046)$. Enfin pour les dimensions "classe» et "activités scolaires" aucune différence significative n'est observée entre $\mathrm{T} 1$ et $\mathrm{T} 2$, (respectivement, $\mathrm{F}(1,556)=1.63, \mathrm{p}=.203$, n.s. et $\mathrm{F}(1,556)=.79$, $\mathrm{p}=.374$, n.s.). 
Tableau 3. Evolution des scores à l'échelle de satisfaction globale, aux résultats scolaires et aux dimensions du questionnaire de bien être en fonction des sessions et du type d'établissement (école primaire vs collège) $(\mathrm{N}=442)$

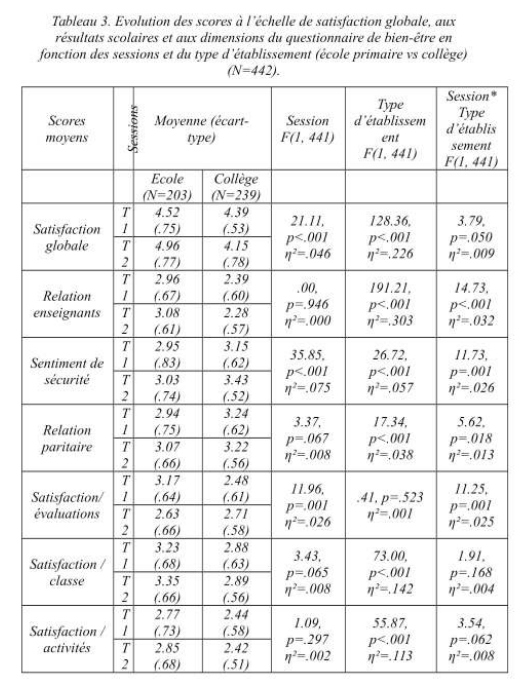

L'étude de l'évolution du bien-être en fonction du type d'établissement (écoles/collèges) porte sur un échantillon de 442 élèves, les 115 élèves scolarisés en CM2 en T1 puis en $6{ }^{\text {ème }}$ en $\mathrm{T} 2$ ayant été exclus des analyses. Les résultats montrent d'une part que la satisfaction scolaire diminue chez les collégiens alors qu'elle augmente chez les écoliers (interaction session*type d'établissement: $\mathrm{F}(1,441)=3.79, \mathrm{p}=.050)$. D'autre part, pour la dimension «relations avec les enseignants », les collégiens ont des appréciations globalement plus négatives que les écoliers et leurs appréciations tendent à devenir plus négatives alors que l'on observe l'inverse pour les écoliers (interaction session*type d'établissement : F $(1,441)=14.73, p=.001)$. S'agissant des dimensions « sentiment de sécurité ", "relations paritaires » et « rapport aux évaluations ", les interactions sont également significatives (respectivement: $\mathrm{F}(1,441)=11.73, \mathrm{p}=.001 ; \mathrm{F}(1,441)=5.62, \mathrm{p}=.018$ et $\mathrm{F}(1,441)=11.25$, $\mathrm{p}=.001$ ). Elles indiquent d'une part que le sentiment de sécurité et la satisfaction à l'égard des relations paritaires des collégiens sont plus élevés que ceux des écoliers, et d'autre part qu'ils augmentent davantage que ceux des écoliers entre les deux temps de mesure. D'autre part, bien que les scores soient plus élevés chez les écoliers que chez les collégiens, le rapport aux évaluations tend à devenir plus négatif pour les écoliers que pour les collégiens. Enfin, pour les dimensions «classe » et "activités scolaires », les interactions ne sont pas significatives. Les résultats indiquent toutefois que les écoliers sont plus satisfaits de leur classe et des activités scolaires que les collégiens. 


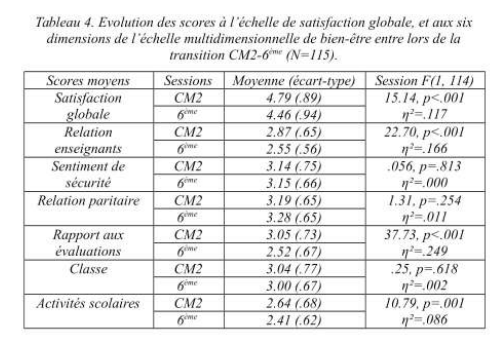

Des analyses identiques (ANOVA à mesures répétées) ont été réalisées sur les mêmes mesures pour les 115 élèves, suivis du CM2 au collège, issus de l'ensemble de l'échantillon. Pour cet échantillon spécifique, on constate également que le score global de satisfaction scolaire a diminué $(\mathrm{F}(1,114)=15.14, \mathrm{p}<.001)$. D'autre part, une diminution des scores est observée pour trois dimensions du questionnaire multidimensionnel : « relations avec les enseignants » $(\mathrm{F}(1,114)=22.70, \mathrm{p}<.001)$, » rapport aux évaluations » $(\mathrm{F}(1,114)=37.73$, $\mathrm{p}<.001)$ et "activités scolaires » $(\mathrm{F}(1.114)=10.79, \mathrm{p}=.001)$. Pour les autres dimensions (sentiment de sécurité, classe et relations paritaires) aucune différence significative n'est n'observée.

\section{Les représentations du bien-être selon les chefs d'établissement}

\section{Analyse du corpus et champs représentationnels}

Les entretiens réalisés avec les chefs d'établissements (directeurs d'école pour le premier degré et principaux de collège pour le second degré) ont été analysés grâce aux statistiques lexicométriques de la méthode $\mathrm{ALCESTE}^{2}$, développée par Reinert (2007). Cette méthode repose sur un découpage répété du vocabulaire utilisé dans le corpus et sur un regroupement de ces mots en "classes " significatives établies en fonction de variables déterminées avant l'analyse du corpus. L'intérêt de ce type d'analyse est de dégager les grands champs représentationnels des discours.

La Classification Descendante Hiérarchique (CDH) appliquée aux entretiens des 17 chefs d'établissements de primaire et de collège a permis de dégager 4 classes prenant en compte $92,02 \%$ des segments classés du texte ${ }^{3}$. Pour chacune de ces 4 classes, nous avons réalisé une analyse de contenu catégorielle thématique en nous appuyant sur le niveau 
sémantique (dégagé par le traitement statistique inférentiel grâce au logiciel), soit la dernière étape de l'analyse de contenu (Dany, 2016).

Figure 1. Classification descendante hiérarchique en primaire et au collège

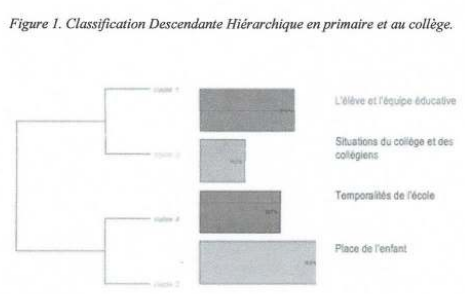

On distingue d'abord deux branches principales du dendrogramme, relativement équivalentes en termes de poids. La première regroupe la classe 1 «L'élève et l'équipe éducative » avec $27,8 \%$ des formes et la classe 2 "Situations du collège et des collégiens " avec $13,2 \%$ des formes. A l'opposé, on trouve les discours regroupés dans les classes 4 «Temporalités de l'école » (23,7\% des formes) et la classe 3 «Place de l'enfant », la plus lourde du corpus $(35,5 \%)$. Une analyse plus détaillée indique que les classes 1 et 2 sont représentatives des discours issus des chefs d'établissements de collège et les classes 3 et 4 de ceux du primaire.

Dans la classe 1, qui regroupe significativement ${ }^{4}$ les discours des chefs d'établissements de collèges publics ZEP et non ZEP, l'élève est au centre. Sa prise en charge est envisagée par les acteurs de l'établissement avec des formes actives telles que: "enseignant", " professeur », " pôle médico-social », " prévention », «CESC » ${ }^{5}$ et « cellule de veille ». La classe 2, également représentative des discours des chefs d'établissement au collège, en particulier ZEP, reflète les contextes géographiques, économiques et sociaux. Elle se rapporte à la situation géographique (le "quartier», la «ville», le «centre») et comporte aussi des formes actives reprenant un discours sur les réseaux, avec des termes tels que « REP » ou « ECLAIR ».

L'autre partie du discours est relative à l'école primaire. La classe 4 «Temporalités de l'école ", caractérise les discours des établissements privés. Les formes actives reflètent l'organisation temporelle, sur une " année », une "journée », pendant les "vacances », etc. La classe 3 "Place de l'enfant» qui caractérise le discours des directeurs d'école primaire est la classe la plus importante de tout le corpus. Les deux formes actives les 
plus significatives sont : "enfant » et «bien-être », ce qui témoigne de préoccupations plus en lien avec le développement affectif, social, de l'enfant, avec des termes tels que " plaisir », « apprendre ", « rire ", "vivre ». De fait, une véritable opposition est à noter entre le collège et l'école primaire, avec un effet de variable des collèges publics et des écoles privées.

L'Analyse Factorielle des Correspondances confirme ces grands champs thématiques mis en évidence, tout en précisant leurs relations de proximité. Le premier facteur $(39,22 \%$ du corpus) sépare logiquement les classes 1 "L'élève et l'équipe éducative " et 2 "Situations du collège et des collégiens », des classes 3 "Temporalités de l'école » et 4 «Place de l'enfant », relatives au primaire. Le second facteur (31,64\%) sépare nettement la classe 1 et la classe 3 . Ces deux facteurs produisent donc sur le graphique trois zones distinctes avec: l'univers de l'élève, son collège et sa prise en charge, zone opposée à deux autres relatives à l'enfant, son bien-être (en correspondance avec le facteur 2 et les difficultés que le collégien peut rencontrer), et le temps qui reflète une représentation des temps de l'enfance, face aux réalités géographiques, sociales, économiques des collégiens.

Figure 2. Analyse factorielle des correspondances (AFC) en primaire et au collège

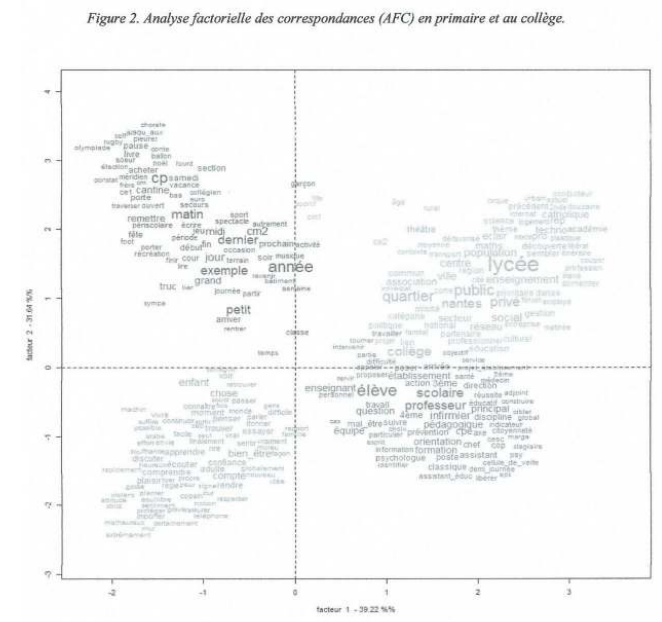

Une opposition dans les représentations et les prises en charge entre école et collège, selon l'environnement

De cette analyse, ressort une opposition entre l'école et le collège, mais également entre l'enfant et l'élève. Ces oppositions conduisent à la fois à une représentation différenciée du public qui fréquente l'établissement et donc à des représentations et des prises en considération différentes du bien-être. En primaire, le bien-être fait partie de la vie quotidienne de l'enfant et de l'école. Il semble "plus simple » à observer et il dissocie 
l'enfant et l'élève. Le terme " enfant » ressort du discours des enseignants de primaire, ce qui n'est pas le cas chez les enseignants de collège. Cette opposition a aussi été soulignée par les sociologues Dubet et Martucelli (1996), qui distinguaient déjà le primaire du secondaire, avec une approche plutôt individuelle en primaire et une approche plus orientée vers le «métier d'élève » en secondaire (Sirota, 1993). En d'autres termes, on observe une distinction en primaire entre les enfants (associés au registre de la famille) et les élèves (associés au domaine professionnel). Au premier abord, cette distinction peut paraître contradictoire dans la mesure où la spécificité des chefs d'établissements de primaire est d'avoir des élèves en face d'eux au quotidien. Mais cette dissociation peut être interprétée dans une perspective développementale en lien à la construction de soi. Comparativement, au collège, l'élève est inscrit dans un fonctionnement plus administratif et organisationnel, autour des temps de l'enfant ${ }^{6}$. Se pose également la question de la proximité avec les enseignants en écho à l'étude quantitative menée auprès des écoliers qui évaluent plus positivement leurs relations avec les enseignants que les collégiens. Plus largement, on observe que les discours des directeurs en primaire sont hétérogènes, plus généraux et centrés sur l'« enfant ».

Comparativement, le discours des chefs d'établissements en collège (davantage le fait de collège ZEP) est moins contrasté et plus diversifié, ce qui illustre la difficulté à appréhender le bien-être. Au collège, c'est le mal-être des élèves plutôt que leur bien-être qui est identifié et les leviers de prévention et de régulation sont inscrits dans une prise en charge collective des actions menées par le CESC. S'il est évoqué, le bien-être est appréhendé selon deux perspectives: l'établissement et l'élève. Un champ représentationnel centré sur l'élève aborde la prise en charge par l'équipe éducative, aux niveaux pédagogiques et institutionnels qui relèvent du projet pédagogique ou du pôle médico-social. Le bien-être et le mal-être sont donc associés aux enseignants, au projet d'établissement, mais aussi au domaine de la santé. En termes contextuels, le collège est décrit comme pris entre école et lycée, avec la préoccupation de créer ou maintenir des liaisons, des partenariats, à la fois dans la transition école/collège (lors des entretiens la réforme du collège et du cycle 3 n'étaient pas d'actualité) mais également au palier d'orientation en $3^{\text {ème }}$, avec les différentes voies d'orientation professionnelles, technologiques, générales. Le poids de l'environnement socio-économique défavorisé et donc celui des élèves est également important. Le bien-être des élèves au collège semble donc inscrit dans son fonctionnement général et irrigue les actions planifiées sur les quatre années, allant de l'accueil des $6^{\text {èmes }}$ à l'orientation des $3^{\text {èmes }}$, ce qui est conforme aux préconisations des projets d'établissements.

Ainsi, il se dégage deux systèmes de représentations du bien-être des élèves : l'un issu des chefs d'établissement en primaire, qui semble aller plutôt de soi, où l'enfant à l'école est considéré comme étant dans le bien-être s'il est heureux de venir à l'école et où les situations de mal-être sont souvent analysées au cas par cas; à l'inverse, les représentations des chefs d'établissement au collège sont plutôt orientées vers une régulation, une anticipation, voire des sanctions dans la perspective de maintenir les collégiens dans le bien-être ou d'éviter le mal-être.

\section{Conclusion et perspectives}

Les données quantitatives récoltées auprès des élèves et les données qualitatives récoltées auprès des chefs d'établissement ont été recueillies de manière concomitante. En accord 
avec les travaux de Creswell et Clark (2007) et de Guérel et Pommier (2012), l'analyse des données a été effectuée à l'aide d'un protocole de recherche par méthode mixte nichée, à partir d'une approche par triangulation méthodologique (Ferrière et al., 2016). Ainsi, les représentations du bien-être des chefs d'établissement ont permis d'étayer les réponses quantitatives des élèves, quant à leur bien-être perçu.

Les résultats longitudinaux montrent que la satisfaction scolaire des élèves diminue significativement avec l'avancée de leur scolarité quel que soit leur type d'établissement (école ou collège). Ces constats vont dans le sens des représentations du bien-être des chefs d'établissement, qui apparaissent plus positives à l'école primaire qu'au collège. De même en considérant l'ensemble de l'échantillon, la satisfaction des élèves vis-à-vis des évaluations scolaires décroit. En effet, au cours de leur scolarité les élèves sont de plus en anxieux envers les évaluations.

Par ailleurs, les élèves d'école primaire déclarent se sentir moins en sécurité dans leur établissement et leurs relations paritaires sont perçues comme étant de moins bonne qualité que celles des collégiens. Ainsi, les écoliers semblent plus sensibles aux phénomènes de harcèlement au sein de leur établissement et sur le trajet de l'école. Ces résultats sont en adéquation avec les discours des directeurs d'école qui ont une vision plus positive du bien-être de leurs élèves, ne considérant que ponctuellement le malaise de certains élèves.

$\mathrm{Au}$ collège, le bien-être décroît significativement en ce qui concerne la satisfaction envers les enseignants. Ces résultats vont dans le sens des analyses qualitatives dans lesquelles le rôle de l'élève est considéré comme un "métier » s'inscrivant dans un fonctionnement plus administratif et organisationnel (Sirota, 1993). Or, il apparaît dans cette étude et dans les recherches internationales que cette dimension contribue à expliquer la satisfaction scolaire des élèves (Epstein, 1981; Verkuyten \& Thijs, 2002; Wigfield et al., 2006 ; Murray-Harvey, 2010 ; Eccles \& Roeser, 2011; OCDE, 2015). De plus, les collégiens sont significativement moins satisfaits de leurs activités scolaires et de leur classe que les écoliers. Ces deux dimensions contribuent également à expliquer la satisfaction scolaire globale des élèves (Currie et al., 2008).

Enfin en se focalisant sur la transition CM2-6 ${ }^{\text {ème }}$, on constate également que le score global de satisfaction scolaire diminue significativement, ainsi que la satisfaction à l'égard des relations avec les enseignants, des évaluations et des activités scolaires. Ces résultats font écho aux différences entre les discours des directeurs d'école et des principaux de collèges. En effet, les premiers ont une vision plus positive que les seconds et cherchent à assurer le bien-être de leurs élèves. A contrario, les principaux de collèges vont plutôt chercher à mettre en place des actions contre le mal-être de leurs élèves. Ces écarts représentationnels recueillis par les entretiens posent la question d'une "culture commune " professionnelle, aussi bien au niveau des professeurs en primaire et en collège que des chefs d'établissement, qui ne disposent pas de la même formation.

Ainsi, pour promouvoir le bien-être des élèves, il conviendrait de développer les pratiques évaluatives encourageantes reflétant plus les efforts des élèves d'école primaire et de collège et qui seraient perçues par les élèves comme moins pénalisantes et décourageantes, comme le suggèrent de longue date les travaux sur les évaluations dans le système scolaire français (cf. par exemple, pour une recension des critiques principales, l'enquête HBSC (2010) ou les interventions et recommandations de la Conférence nationale de l'évaluation organisée par le Ministère de l'éducation nationale les 11 et 12 décembre 2014 à Paris $^{7}$ ). A l'école primaire, offrir des lieux d'apprentissage sécurisants et 
prévenir collectivement de l'intimidation ou de comportements inappropriés, en développant les compétences sociales des élèves (Florin, 2011), pourrait permettre d'améliorer le bien-être des élèves à l'école. Au collège, promouvoir et encourager les initiatives et les comportements positifs des élèves, mais aussi développer des actions pédagogiques différenciées, adaptées aux besoins de chaque élève, favoriseraient le développement de relations de qualité avec leur enseignant et une meilleure réussite dans les apprentissages, comme le préconise le rapport Innocenti 13 de l'UNICEF (2016). Afin d'améliorer leurs relations avec les autres élèves, mais aussi avec les enseignants, habituer les élèves à travailler en groupe, en binôme ou dans des relations de tutorat pourrait également contribuer à ce qu'ils se sentent mieux à l'école. Néanmoins, pour que ce tutorat soit efficace, l'enseignant doit le mettre place de manière très structurée et dans les situations d'apprentissage précises (Topping, 1996).

Ces préconisations rejoignent les recommandations internationales et nationales sur les pratiques de promotion et de prévention les plus efficaces pour favoriser le bien-être des élèves (Palluy \& Laverdure, 2010; Debardieux, 2011; Broussouloux, Berger, Bristol, \& Morand, 2014), mais aussi certains termes de la loi de 2013 pour la refondation de l'école. Par ailleurs, la mise en place du nouveau cycle 3 (reliant désormais les deux dernières années de l'école primaire et la première année du collège) pourrait permettre une meilleure transition entre l'école primaire et le collège (Bulletin Officiel, novembre 2015). Néanmoins, comme le souligne Prost (2013), le succès d'une réforme éducative est déterminé par de nombreux facteurs et suppose l'adhésion des enseignants et des parents d'élèves via des concertations au sein des établissements. Les données de cette recherche concernant les enfants ont été recueillies par auto-évaluation directement auprès d'eux, grâce aux questionnaires qu'ils ont renseignés et complétés par des entretiens auprès des chefs d'établissement. Une troisième année de collecte de données est en cours, qui permettra d'étudier l'évolution à plus long terme de la relation entre bien-être et performances scolaires, mais aussi de prendre en compte l'évolution d'autres dimensions du bien-être étudiées à partir de la $2^{\text {ème }}$ année: sentiment d'appartenance à l'établissement, autonomie et droits des élèves et perception des différents lieux et aménagements scolaires. Ces données collectées durant cette troisième année, liées entre autres au rôle et à la place de l'élève dans son cadre scolaire, permettront de mieux saisir le vécu des élèves au sein de celui-ci comme lieu de vie, de "vivre ensemble » et de rendre compte de relations de causalité avec d'autres variables (interindividuelles, sociofamiliales et du bien-être à l'école et au collège) mesurées en T1. Ainsi, les résultats recueillis dans une perspective multidimensionnelles et par triangulation permettront, par croisements et confrontation, de proposer des préconisations à destination des équipes éducatives afin de promouvoir le bien-être des élèves, à l'école et au collège, pour prévenir et lutter contre le mal-être de certains d'entre eux, mais aussi de mieux les préparer au changement d'établissement lors de la transition école primaire-collège notamment. En effet, le questionnaire Be-Scol peut être utile aux équipes éducatives pour leur permettre d'identifier plus facilement les points faibles et les points forts de l'établissement, et de ce fait, pouvoir agir en conséquence (Debarbieux \& Blaya, 2009; Wilson \& Lipsey, 2006; MEN, 2013). Plus précisément, élaborer une démarche de concertation avec l'ensemble du personnel de l'établissement permettrait une représentation partagée des différentes dimensions du bien-être des élèves, mais aussi une élaboration d'actions ciblées sur des points à améliorer, en collaboration avec les élèves ; ce travail d'équipe favorisait également le développement d'un climat scolaire de bonne qualité au sein de l'établissement (MEN, 2013) pour lequel des outils sont 
disponibles, sur plusieurs sites (Eduscol, sites académiques, etc.). Par ailleurs, les représentations différenciées du bien-être des chefs d'établissements montrent l'intérêt de sensibiliser les futurs enseignants de premier et second degré, lors de formations communes, à l'importance de prendre en compte la qualité de vie de leurs élèves à l'école et au collège, dans l'objectif d'avoir une représentation partagée du bien-être à l'école et au collège, et de l'évolution des besoins des enfants dans leur lieu de vie scolaire au cours de leur développement.

\section{BIBLIOGRAPHIE}

Afsa, C. (2015). Où fait-il bon enseigner ? Education et Formations, 88-89, 61-77.

Bacro, F., Florin, A., Guimard, P., \& Rambaud, A. (2013). L'évaluation du bien-être des jeunes enfants : nouvelles perspectives en psychologie du développement. In A. Florin, M. Préau (Eds.), Le bien-être (p. 71-82). Paris: L'Harmattan.

Bacro, F., Rambaud, A., Florin, A., \& Guimard, P. (2011). L'évaluation de la qualité de vie et son utilité dans le champ de l'éducation. ANAE, 112-113, 189-194.

Bavoux, P., \& Pugin, V. (2013). Baromètre annuel du rapport à l'école des enfants de quartiers populaires . AFEV.

Billaudeau, N., \& Vercambre-Jacqhot, M.-N. (2015). Satisfaction professionnelle des enseignants du secondaire. Quelles différences entre public et privé ? Education et Formations, 88-89, 201-220.

Broussouloux, S., Berger, D., Bristol, P., \& Morand, J. (2014). Promouvoir la santé des élèves dans les établissements scolaires. INPES, en ligne.

Bulletin Officiel du MEN, $n^{\circ}$ 11, 26 novembre 2015.

Creswell, J. W., \& Clark, V. L. P. (2007). Designing and conducting mixed methods research. Australian and New Zealand Journal of Public Health, 31(4), 303-396.

Currie C., Zanotti, C., Morgan, A., Currie, D., De Looze, M., Roberts, C., Samdal, O., Smith, O. R.F., \& Barnekow, V. (2012). Social determinants of health and well-being among young people. Health Behaviour in School-aged Children (HBSC) study: international report from the 2009/2010 survey. Copenhagen, WHO Regional Office for Europe, 2012 (Health Policy for Children and Adolescents, No. 6).

Dany, L. (2016). Analyse qualitative du contenu des représentations sociales. In G. Lo Monaco, S. Delouvée et P. Rateau (Eds), Les représentations sociales. Théories, méthodes et applications (p. 85-102). Bruxelles : De Boeck.

Debarbieux, E. (2011). À l'école des enfants heureux... enfin presque.... Paris, Observatoire international de la violence à l'école : Unicef.

Debarbieux, E., \& Blaya, C. (2009). Le contexte et la raison : agir contre la violence à l'école par « l'évidence »? in Criminologie. Criminologie, 42(1), 13-31. 
Durlak, J.A., Weissberg, R.P., Dymnicki, A.B., Taylor, R.D., \& Schellinger, K.B. (2011). The impact of enhancing students' social and emotional learning: a meta-analysis of school-based universal interventions. Child Development, 82, 405-32.

Dubet, F., \& Martucelli, D. (1996). A l'école : sociologie de l'expérience scolaire. Paris: Seuil.

Eccles, J.S., \& Roeser, R.W. (2011). Schools as Developmental Contexts During Adolescence. Journal of Research on Adolescence, 21(1), 225-241.

Epstein, J.L. (1981). The Quality of School Life. Lexington Books/D. C. Heath, Lexington.

Ferrière, S. (2013). L'ennui à l'école primaire : Représentations Sociales, Usages et Utilités. Paris : L'Harmattan.

Ferrière, S., Bacro, F., Florin, A., \& Guimard, P. (2016). Le bien-être en contexte scolaire : Intérêt d'une approche par triangulation méthodologique. Cahiers Internationaux de Psychologie Sociale, 3 (111), 341-365.

Florin, A. (2011). Des apprentissages fondamentaux aux compétences pour demain : les apports de la psychologie de l'éducation. Bulletin de Psychologie, 64(1), n 511, 15-29.

Gill, S. (2009). Well-being in education: a conceptual framework, Working Paper, European Consortium of Learning For Well-Being.

Guével, M.-R., \& Pommier, J. (2012). Recherche par les méthodes mixtes en santé publique : enjeux et illustration. Santé Publique, 24(1), 23-38.

Guimard, P., Bacro, F., \& Florin, A. (2013). Évaluer le bien-être à l'école et au collège. In F. Bacro (Eds.), La qualité de vie : approches psychologiques (p. 45-64). Rennes : PUR.

Guimard, P., Bacro, F., \& Florin, A. (2014). Évaluer la satisfaction scolaire et le bien-être des élèves à l'école et au collège. In P. Guimard, \& C. Sellenet (Eds.), Evaluation des besoins des enfants et qualité de vie : regards croisés France-Canada (p. 1-25). Paris : L'Harmattan.

Guimard, P., Bacro, F., Ferrière, S., Florin, A., Gaudonville, T., \& Ngo, H. T. (2015). Le bien-être des élèves à l'école et au collège. Validation d'une échelle multidimensionnelle, analyses descriptives et différentielles. Education \& formations, 88-89, 163-184.

Harter, S. (1982). The Perceived Competence Scale for Children, Child Development, 53(1), 87-97.

Hoffman, D.M. (2009). Reflecting on social emotional earning: A critical perspective on trends in the United States. Review of Educational Research, 79(2), 533-556.

Huebner, E.S., Ash, C., \& Laughlin, J.E. (2001). Life experiences, locus of control, and school satisfaction in adolescence. Social Indicators Research, 55, 167-183.

Huebner, E.S., Zullig, K.J., \& Saha, R. (2012). Factor Structure and Reliability of an Abbreviated Version of the Multidimensional Students' Life Satisfaction Scale. Child Indicators Research, 5(4), 651-657.

Lester, L., \& Cross, D. (2015). The Relationship Between School Climate and Mental and Emotional Wellbeing Over the Transition from Primary to Secondary School. Psychological Well-Being, 5(9), 1-15.

Loi n 2013-595 du 8 juillet 2013 d'orientation et de programmation pour la refondation de l'école de la République.

Long, R.F., Huebner, E.S., Wedell, D.H., \& Hills, K.J. (2012). Measuring School-Related Subjective Well-Being in Adolescents. American Journal of Orthopsychiatry, 82(1), 50-60. 
MEN (Ministère de l'Education Nationale). (2013). Agir sur le climat scolaire à l'école primaire. http://eduscol.education.fr

Murray-Harvey, R. (2010). Relationship influences on students' academic achievement, psychological health and well-being at school. Educational and Child Psychology, 27(1), 104-115.

OCDE (2013). Regards sur l'éducation 2013 : les indicateurs de l'OCDE. Paris : OCDE.

OCDE (2015). PISA à la loupe. Paris : OCDE.

Palluy, J., \& Laverdure, J. (2010). Réussite éducative, santé, bien-être : agir efficacement en contexte scolaire : synthèse de recommandations. Institut national de santé publique du Québec.

Pierrehumbert, B., Plancherel, B., \& Jankech-Caretta, C. (1987). Image de soi et perception des compétences propres chez l'enfant : Présentation d'un questionnaire récent d'estime de soi pour enfants. Revue de Psychologie Appliquée, 37(4), 359-377.

Pollard, E.L., \& Lee, P.D. (2003). Child Well-being: A Systematic Review of the Literature. Social Indicators Research, 61(1), 59-78.

Prost, A. (2013). Du changement dans l'école. Les réformes de l'éducation de 1936 à nos jours. Paris: Seuil. Randolph, J.J., Kangas, M., \& Ruokamo, H. (2009). The Preliminary Development of the Children's Overall Satisfaction with Schooling Scale (COSSS). Child Indicator Resarch, 2(1), 79-93.

Randolph, J.J., Kangas, M., \& Ruokamo, H. (2010). Predictors of Dutch and Finnish Children's Satisfaction with Schooling. Journal of Happiness Study, 11(2), 193-204.

Ratinaud, P. (2009). IRaMuTeQ : Interface de R pour les Analyses Multidimensionnelles de Textes et de Questionnaires. http://www.iramuteq.org

Reinert, M. (2007). Postures énonciatives et mondes lexicaux stabilisés en analyse statistique de discours. Langage et Société, 3, 189-202.

Shankland, R., \& Rosset, E. (2016). Review of Brief School-Based Positive Psychological Interventions: A Taster for Teachers and Educators. Educational Psychology Review, 27(4), 1-30.

Sirota, R. (1993). Le métier d'élève. Revue française de pédagogie, 1(104), 85-108.

Topping, K. J. (1996). The effectiveness of peer tutoring in further and higher education: A typology and review of the literature. Higher education, 32(3), 321-345.

UNICEF (2016). Bilan Innocenti 13. Equité entre les enfants, tableau de classement des inégalités de bien-être entre les enfants des pays riches. www.unicef.fr

Verkuyten, M., \& Thijs, J. (2002). School Satisfaction of Elementary School Children: The Role of Performance, Peer Relations, Ethnicity and Gender. Social Indicators Research, 59(2), 203-228.

Wilson S., \& Lipsey M. W. (2006). The Effectiveness of School-Based Violence Prevention Programs for Reducing Disruptive and Aggressive Behavior: A Meta-analysis. International Journal on Violence in Schools, 1, 2-26.

Wigfield, A., Eccles, J.S., Schiefele, U., Roeser, R., \& Davis-Kean, P. (2006). Motivation. In N. Eisenberg (Ed.), Handbook of child psychology (Vol. 3, 6th ed., pp. 933-1002). New York: Wiley.

Zullig, K.J., Koopman, T.M., \& Huebner, E.S. (2009). Beyond GPA: Towards more comprehensive assessments of students' school experiences. Child Indicators Research, 2, 95-108.

Annexe 1. Echelle de satisfaction scolaire globale (Huebner et al., 2012 ; Guimard et al., 2015)

Annexe 2. Questionnaire Be-Scol (Guimard et al., 2015) 


\section{NOTES}

1. Etude réalisée dans le cadre d'une convention financée par la Direction de l'Evaluation, de la Prospective et de la Performance, le Défenseur des Droits et le Commissariat général à l'égalité des territoires.

2. Nous avons utilisé le logiciel libre de lexicométrie Iramuteq (Ratinaud, 2009), implémentation libre d'ALCESTE qui permet, par la méthode d'analyse hiérarchique descendante (CDH), de générer des « classes » en reproduisant la méthode de classification développée par Reinert.

3. Le corpus soumis au traitement lexicométrique est composé d' "unités de contexte initiales » ou u.ci.. et d' " unités de contexte élémentaires » ou u.c.e. Les u.c.i. sont les divisions considérées comme naturelles du corpus, dans notre cas il s'agit des 17 entretiens. Ces u.c.i. comprennent également des « variables étoilées " relatives aux caractéristiques que les chercheurs souhaitent prendre en compte dans l'analyse globale, souvent liées à l'échantillonnage. Nous avons donc pour chaque entretien spécifié s'il s'agit d'un chef d'établissement en primaire ou au collège et la strate de l'établissement (privé, public ZEP et public non ZEP).

4. Les «variables signalées » selon l'établissement (primaire vs collège) et la strate (privé vs public ZEP vs public non ZEP) contribuent plus ou moins significativement au discours d'une classe en fonction du test du Chi2 calculé selon un coefficient d'association d'une forme lexicale.

5. Le CESC (Comité d'éducation à la santé et la citoyenneté), spécifique au second degré et inscrit dans le projet d'établissement, a pour objectifs d'aborder diverses problématiques éducatives : l'éducation à la citoyenneté, à la santé et à la sexualité la prévention de la violence et des comportements à risque, etc.

6. Les entretiens ont été réalisés lors des changements de rythmes scolaires, ce qui peut expliquer l'importance des préoccupations organisationnelles.

7. http://www.conference-evaluation-des-eleves.education.gouv.fr/consultez-les-expertises

\section{RÉSUMÉS}

Cet article vise à repérer quelques actions susceptibles de promouvoir le bien-être à l'école et au collège en partant du point de vue des élèves et des chefs d'établissements. Il s'appuie sur les données d'une étude longitudinale de 2 années réalisée auprès de 557 élèves scolarisés à l'école primaire et au collège ayant renseigné un questionnaire multidimensionnel de bien-être à l'école ainsi que sur les réponses de chefs d'établissement à un entretien semi-directif. Les résultats montrent que le bien-être des élèves décroit dans plusieurs dimensions, notamment lors du passage $\mathrm{CM} 2 / 6^{\text {ème, }}$ et que les représentations du bien-être des chefs d'établissement diffèrent selon le type d'établissement. Des préconisations d'amélioration du bien-être des élèves sont envisagées en tenant compte des apports de la littérature internationale.

This article aims to identify some actions to promote well-being in primary and secondary school, starting from the own perspective of students and principals. It draws on data from two years' longitudinal study conducted among 557 students attending primary and secondary school who completed a multidimensional questionnaire of well-being at school and on the responses of headteachers to a semi-structured interview. The results show that the well-being of students 
decreased in several dimensions particularly during the transition from elementary to secondary school and that principals' representations of well-being differ depending on the type of establishment. The recommendations to improve the well-being of pupils are envisaged taking into account the contributions of the international literature.

INDEX

Keywords : well-being perceived, school satisfaction, longitudinal study, primary school, middle school, methodological triangulation

Mots-clés : bien-être perçu, satisfaction scolaire, étude longitudinale, école primaire, collège, triangulation méthodologique

\section{AUTEURS}

\section{TIPHAINE GAUDONVILLE}

Université de Nantes, CREN (Centre de Recherche en Education de Nantes, EA 2661) 